\title{
El camino del Uruguay hacia la diversificación de su matriz eléctrica - alianzas, interpretaciones y controversias
}

\section{Uruguay's road towards diversification of its electricity matrix - alliances, interpretations and controversies}

ARTÍCULO

\author{
Guillermo Jorge \\ Universidad Nacional de General Sarmiento, Argentina. Contacto: gjorge@campus.ungs.edu.ar
}

Recibido: abril de 2021

Aceptado: mayo de 2021

\section{Resumen}

En este trabajo se analiza el camino que ha tomado Uruguay a partir del año 2005 para diversificar su matriz eléctrica y hacer frente a las diferentes crisis y tensiones que estresaban su sistema eléctrico. La dependencia del precio internacional del crudo, la exposición a estacionalidades de corto y largo plazo en la producción hídrica, la desinversión y falta de participación estatal anteriores al 2005 llevaron al planteo de una política de expansión a corto, mediano y largo plazo con la cual el Uruguay se pudo posicionar como líder regional en fuentes de generación alternativas. Analizamos en el marco teórico de los sistemas socio-técnicos las alianzas que posibilitaron este desarrollo y analizamos las controversias que se generaron en torno al precio de la energía y al rol del estado como garante de la inversión privada mediante mecanismos financieros de compensación.

Palabras claves: Uruguay, energías renovables, matriz eléctrica, sistemas socio-técnicos.

\begin{abstract}
This paper analyzes the path that Uruguay has taken since 2005 to diversify its electricity matrix and face the different crises and tensions that stressed its electricity system. Dependence on the international price of crude oil, exposure to short and long-term seasonality in hydropower production, disinvestment and lack of state participation prior to 2005 led to the proposal of a short, medium and long-term expansion policy with which Uruguay was able to position itself as a regional leader in alternative generation sources. We analyze in the theoretical framework of sociotechnical systems the alliances that made this development possible and we analyze the controversies that were generated around the price of energy and the role of the state as guarantor of private investment through financial compensation mechanisms.
\end{abstract}


Keywords: Uruguay, renewable energies, electricity matrix, socio-technical systems.

\section{1 - Introducción}

El caso del desarrollo energético uruguayo es por demás interesante, ya que ha podido en el transcurso de sólo unos pocos años pasar de ser un país con una matriz eléctrica restringida y básicamente bivariada, con incidencia principalmente de la generación fósil e hídrica, a ser uno de los países líderes en la región en energías renovables y poseer una matriz diversificada con incidencia de renovables como la biomasa, la energía eólica y la solar fotovoltaica. ${ }^{1}$

Este cambio fue realizado en un período que comienza con la asunción del presidente Tabaré Vázquez en el año 2005, extendiéndose por un período de 15 años en los cuales se consolidó el desarrollo de fuentes alternativas de generación. Para visualizar este proceso de cambio tecnológico, podemos mirar las instantáneas de la matriz uruguaya antes y después de andar el camino de desarrollo. En la figura 1 (paneles superiores) observamos la potencia instalada (en porcentaje de participación) para los años 1999 y 2019 (MIEM-Datos abiertos, 2021). Se observa hacia 1999 básicamente dos formas de producción preponderantes, la generación térmica alimentada por combustible fósil (26\%) y la generación hídrica que provenía de sus cuatro centrales hidroeléctricas (una de ellas compartida con Argentina). El cambio hacia 2019, 20 años después, es notable. La energía eólica se convierte en la segunda fuente después de la hídrica con $31 \%$, la solar fotovoltaica comienza a ganar fuerza, entra en jeugo la energía por biomasa, y se observa en general un equilibrio bien logrado entre todas las fuentes.

Si bien la potencia instalada fósil continua representando aproximadamente un cuarto del total, esta potencia tiene hoy en día una finalidad de reserva fundamentalmente, para compensar variaciones temporales cortas de las otras fuentes (intermitencias o estacionalidades). Si tomamos la participación de dicha fuente en la generación de energía eléctrica) entre 2008 (período en donde tuvo un pico de generación) y el año 2018 (figura 1, paneles inferiores), vemos que en diez años pasó del $38 \%$ a tan sólo el 3\%, también por supuesto aumentando la participación de la eólica y solar que absorben esta energía fósil no producida. Esta disminución drástica de la generación fósil permitió otorgarle mayor independencia técnica y financiera, y reducir la emisión de contaminantes atmosféricos.

Uruguay se convierte entonces en un caso paradigmático de estudio, que estudiaremos desde un abordaje socio-técnico, el cual nos permitirá seguir los caminos de desarrollo y develar la trama de alianzas que lo posibilitaron, y también contrastar las diferentes posiciones

\footnotetext{
${ }^{1}$ El Uruguay es un país pequeño en relación a sus vecinos, y comparte muchas características geofísicas con la zona sur del Brasil y la zona pampeana argentina. Su escala territorial y poblacional es en relación 1/2 de Chile, 1/12 de Argentina y 1/50 del Brasil. Se conforma como un estado unitario presidencialista, con división política en departamentos que no poseen autonomía.
} 
controvertidas del resultado final del proceso, con un país rico en energía, pero en energía costosa para la población.

URUGUAY: MATRIZ ELÉCTRICA
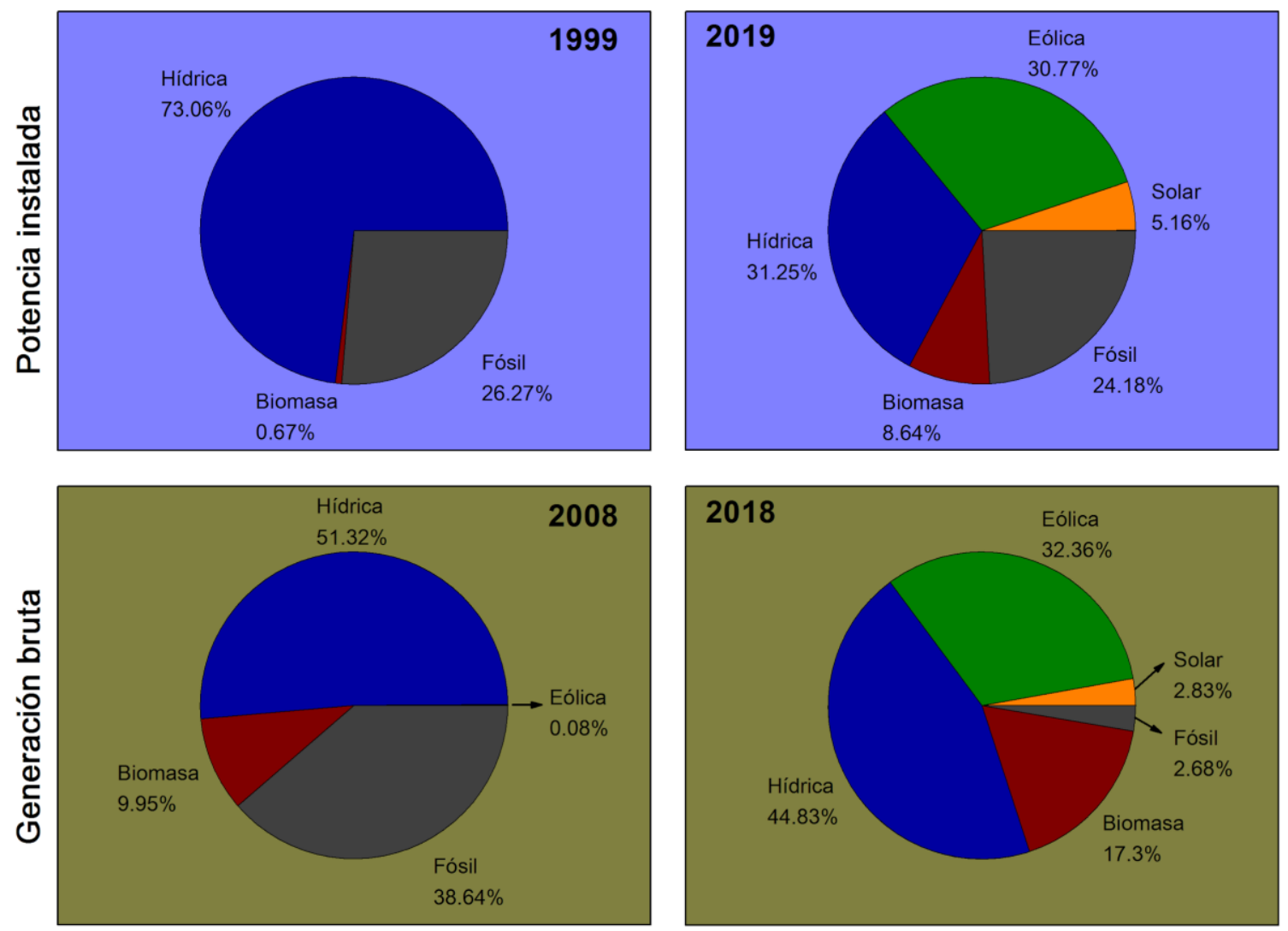

Figura 1: Matriz eléctrica del Uruguay. Se observa en los dos cuadros superiores la potencia instalada por fuente en porcentaje para 1999 y 2019. En los cuadros inferiores se muestra la generación eléctrica anual bruta en porcentaje del año 2008 y 2018. Fuente: elaboración propia en base a datos de MIEM-Datos abiertos (2021).

Si bien el desarrollo de energías alternativas en Uruguay es un caso de estudio muy interesante, no son muy numerosos los trabajos dedicados a su análisis. Los aspectos técnicos del desarrollo se han descrito en algunos informes, como los de la Agencia Internacional de Energías Renovables sobre el estado del arte en la materia (IRENA, 2016a), y un resumen ejecutivo de las políticas en energías renovables del país (IRENA, 2016b). También el Observatorio de Energía Renovable para América Latina produjo un informe completo sobre la situación de las energías renovables en Uruguay (Caldas, 2011), mientras que la Dirección Nacional de Energía, la Dirección Nacional de Medio Ambiente y la Agencia Española de Cooperación Internacional para el Desarrollo en Uruguay publicó un informe sobre la consultoría Medio Ambiente y Energía en Uruguay (DNE-DINAMA, 2013). Se ha realizado también un trabajo de análisis del plan energético uruguayo de largo plazo (González y Méndez, 2015). Por su parte, 
se pueden encontrar los informes oficiales de la consultoría de energía del Plan Estratégico Nacional en Ciencia, Tecnología e Innovación (Méndez, 2008). Por otra parte, se ha estudiado desde el marco de la interfaz Ciencia Política, entendida como los procesos sociales que llevan a la generación de espacios de inter-relacionamiento entre científicos y otros actores, los ámbitos de diálogo entre instituciones científico-técnicas y los tomadores de decisiones que fueron fundamentales en el desarrollo eólico uruguayo (Bernardi, 2019). En cuanto a estudios desde la Construcción Social de la Tecnología, se han realizado análisis enfocados en el desarrollo particularmente eólico, estudiando el flujo de conocimiento enmarcado en la teoría del materialismo cognitivo (Ardanche, 2016; Ardanche, 2017a). Los autores de estos trabajos también han analizado el desarrollo eólico en el marco teórico de las transiciones tecnológicas y los sistemas de innovación (Ardanche, 2017b). Anteriormente hemos estudiado el proceso de desarrollo eólico desde el marco de los sistemas socio-técnicos (Jorge, 2020). Este trabajo entonces tiene por objetivo contribuir al estudio y análisis socio-técnico del peculiar desarrollo energético que tuvo lugar en el Uruguay, completando y abarcando a las diversas formas de producción alternativas incluyendo la solar, y desarrollando las alianzas configuradas, y las controversias que se generaron durante el proceso y que aún hoy no han tenido una clausura definitiva.

Para el desarrollo del trabajo se procedió de acuerdo a la siguiente metodología. Primeramente se identificaron los hitos históricos de desarrollo energético, construyéndose una cronología. Se utilizaron también diversos indicadores cuantitativos para analizar la evolución temporal del sistema eléctrico (potencia instalada, generación bruta anual según fuente, evolución de precios de la energía y del petróleo), obtenidos de diversas fuentes bibliográficas técnicas. Sobre esta base se analizaron los procesos de cambio que transcurrieron a partir del año 2005, utilizándose como marco teórico principal las conceptualizaciones provenientes la teoría de la construcción social de la tecnología. Se identificaron los actores y grupos sociales relevantes y sus roles dentro del desarrollo. En base a estas identificaciones se reconstruyeron las alianzas socio-técnicas que intervinieron en el desarrollo. Se analizó también desde este marco teórico las principales controversias del caso y su estado actual. Se utilizaron fuentes de información cualitativas, en base a artículos técnicos, artículos especializados, información oficial y notas de prensa.

El trabajo se organiza de la siguiente manera: en la próxima sección presentaremos los lineamientos teóricos que enmarcan este estudio. En la sección 3 profundizaremos en el proceso de desarrollo energético llevado a cabo en el país. En la sección 4 investigaremos las diferentes alianzas conformadas que allanaron el camino hacia la diversificación. En la sección 5 estableceremos las diferentes interpretaciones de la energía eléctrica que se configuran socialmente. En la sección 6 se discutirán diversas controversias que se fueron generando en el proceso. Finalmente presentaremos las conclusiones en la sección 7 . 


\section{2 - Lineamientos teóricos}

En este trabajo adoptaremos conceptualizaciones provenientes de la Construcción Social de la Tecnología, que nos servirán para analizar el tejido tecno-social del proceso de desarrollo (Hughes, 1986; Pinch y Bijker, 2008). En particular se puede mencionar el concepto de grupos sociales relevantes, como aquellos grupos que construyen el artefacto tecnológico y sus procesos asociados; y el concepto de flexibilidad interpretativa que nos habla de los sentidos que los grupos otorgan al artefacto tecnológico.

Cuando la flexibilidad interpretativa de los diferentes grupos es divergente y no homogénea, pueden darse procesos de estabilización y clausura. En el proceso de estabilización, disminuye la divergencia de sentidos o flexibilidad, surgiendo una interpretación más homogénea dentro de los diferentes grupos sociales. En la clausura, la interpretación dominante pasa a ser la interpretación consensuada y la divergencia se cierra en favor de ella. Puede darse el caso de que no se logre una clausura definitiva, pero sí una estabilización en donde pueden darse funcionamientos múltiples para distintos grupos sociales.

Para el estudio de la producción de tecnologías en países de América Latina se han desarrollado modelos teóricos que resultan especialmente útiles a la hora de estudiar y analizar los procesos socio-técnicos que determinan un artefacto tecnológico (Thomas, 2008). Para estos análisis se utilizan algunas conceptualizaciones muy útiles: las dinámicas socio-técnicas nos describen el conjunto de interacciones técnicas, económicas, sociales, y políticas que determinan el desarrollo técnico. Las trayectorias socio-técnicas son los procesos de aprendizaje, construcción de funcionamientos, estrategias y políticas que se desarrollan dentro de una o varias dinámicas. Las alianzas socio-técnicas son las articulaciones entre los elementos intervinientes en el desarrollo, que pueden ser desde actores o grupos sociales, tecnologías, conocimiento, recursos, regulaciones, instituciones, condicionamientos, etc., que conforman coaliciones que son las que posibilitan o alientan el desarrollo o lo entorpecen. Las alianzas permiten analizar relaciones de poder y determinar los actores traccionantes en el proceso. Vamos a tomar este concepto en forma central en nuestro análisis.

Existen también algunas especificidades detectadas por algunos autores, referidas al estudio de desarrollos en sistemas energéticos, en particular nos sirve para el estudio del sistema eléctrico. Mencionaremos aquí dos: el problema de la invisibilidad, y el de la multiplicidad interpretativa. El primer problema consiste en conceptualizar la forma en que la mayoría de la gente percibe al sistema eléctrico, como un sistema invisible. La mayoría de la gente se da cuenta de la importancia del sistema eléctrico cuando falla (Edwards, citado en Sovacool, 2019). El sistema se vuelve invisible en situaciones normales. El problema aquí es que en los momentos de estabilidad del sistema la invisibilidad genera una reducción en la presión social hacia el cambio, que sólo se produce cuando el sistema falla y ya es tarde. Este factor es clave para entender la flexibilidad interpretativa de una tecnología. 
El segundo problema, el de la multiplicidad interpretativa, ligado muy fuertemente al concepto de flexibilidad, nos habla de los cinco significados distintos de la palabra energía, en términos de sus representaciones sociales (Stern y Aronson, citados en Sovacool, 2009). La visión científica sostiene que la energía es la capacidad de realizar trabajo en un sistema y puede manifestarse de diferentes formas. La visión económica, en donde la energía es una mercancía que cotiza en un mercado de compra-venta (una commodity). La visión ecológica, que enfatiza los valores de sustentabilidad en la producción y uso. La visión de bienestar social que sostiene el acceso a la energía como derecho humano básico. Finalmente la visión de seguridad que se focaliza en la estabilidad política y disponibilidad de los recursos.

Los lineamientos teóricos utilizados a lo largo de este trabajo abarcan las diferentes conceptualizaciones del enfoque socio-técnico, en especial en torno a la flexibilidad interpretativa y a las alianzas socio-técnicas, integradas con las problemáticas específicas mencionadas, que nos parecen esenciales a la hora del estudio del desarrollo eléctrico uruguayo.

\section{3 - El camino hacia la diversificación}

El año 2005 fue un quiebre en la política uruguaya. Era la primera vez que un partido no tradicional (Partido Nacional o Blanco y Partido Colorado), en este caso el Frente Amplio, llegaba al poder y con mayoría propia. En las elecciones del año 2004 la alianza denominada Encuentro Progresista - Frente Amplio - Nueva Mayoría obtuvo el 50,5\% de los votos. El $1^{\text {ro }}$ de Marzo de 2005 asumió Tabaré Vázquez su primera presidencia (Buquet y Chasquetti, 2005).

Las políticas públicas emprendidas por sus antecesores desde la recuperación de la democracia en 1985, y en especial desde el año $1990,{ }^{2}$ estuvieron enmarcadas en los movimientos neo-liberales de la época, otorgándoles una orientación destinada sobre todo al mercado como principal regulador y traccionante del desarrollo económico y social. La ley de Empresas Públicas (Ley 16.211) del año 1991 fue implementada para el ingreso de capital privado en algunas empresas y la privatización de otras tantas. En el caso particular de las empresas energéticas estatales, UTE (Usinas y Transmisiones Eléctricas, la empresa eléctrica estatal) y ANCAP (Administración Nacional de Combustibles, Alcohol y Portland, la empresa de combustibles estatal) no perdían su condición de monopólicas pero se permitía el ingreso de capital privado, en el caso del mercado eléctrico particularmente en el proceso de generación y transmisión de energía eléctrica. La ley fue parcialmente derogada en un referéndum al año siguiente, pero los artículos derogados se referían principalmente a la privatización de la empresa telefónica estatal ANTEL, con lo cual todos los artículos referidos al mercado eléctrico se

\footnotetext{
2 Julio María Sanguinetti del Partido Colorado 1985-1990. Luis Alberto Lacalle del Partido Nacional 1990-1995. J. M. Sanguinetti (segundo mandato) 1995-2000. Jorge Battle del Partido Colorado 2000 2005.
} 
mantuvieron vigentes. También por la vía del referéndum se revocó, más adelante, una ley que intentaba remover el monopolio de ANCAP sobre el mercado de combustibles (ley 17.448).

En el año 1997 se aprueba el marco regulatorio del sector eléctrico por ley 16.832, el cual crea la Unidad Reguladora de la Energía Eléctrica (UREE, más tarde denominado URSEA: Unidad Reguladora de Servicios de Energía y Agua, por ley 17.598), que tiene por objetivo la fiscalización del mercado, control de calidad y seguridad de servicios y la defensa del consumidor. También se crea la Administradora del Mercado Eléctrico (ADME) que tiene a su cargo el despacho nacional de cargas. Esta ley también estableció lo que se conoce como libertad de generación, exceptuando a dicho proceso del carácter de servicio público que tenían los tres procesos energéticos (generación, transmisión y distribución) desde la sanción de la ley nacional de electricidad del año 1977. De este modo, aunque se mantiene el monopolio de UTE sobre la transmisión y la distribución, el proceso de generación queda abierto a la entrada de actores privados que por su cuenta, o asociados a la empresa estatal, quieran participar en la generación eléctrica siguiendo las normativas específicas e integrados al despacho de cargas. Las diferentes reglamentaciones mencionadas pueden ser consultadas en MIEM-Normativa (2021).

Más allá de las reglamentaciones y normativas específicas dictadas con anterioridad al cambio de gobierno de 2005, las diferentes administraciones evidenciaban una tendencia a desacoplar la actividad de las empresas públicas del sector con las dependencias ejecutivas de gestión, el Ministerio de Energía y Minería (MIEM) y su Dirección Nacional de Energía (DNE). Esta falta de coordinación de políticas limitaba la presencia del estado y su capacidad de iniciativa, dejando en manos del mercado el proceso de desarrollo. Las plantas técnicas de la gestión energética estaban muy disminuidas, y la relación con las empresas estatales (entes autónomos) estaba muy debilitada. La política se centraba sólo en la tercerización de servicios y la desregulación de actividades (González y Méndez, 2015).

En cierta medida, la tarea que se encomendó la nueva administración es generar un cambio de paradigma para la política energética: pasar de un modelo de mercado a un modelo socialdemócrata, en donde el estado debería ser el eje del desarrollo energético. Con este criterio y objetivo principal en mente, se gestó la Política Energética Uruguay 2030 (PEU2030, ver MIEMNormativa, 2021) que fuera aprobada en el año 2008. Este documento define la política energética del país a largo plazo sobre cuatro ejes principales: oferta, demanda, social e institucional.

En el eje institucional, se encomienda al estado ser el principal traccionador del sistema, diseñando, planificando y coordinando todas las políticas energéticas a través de la DNE. Impone a las empresas públicas UTE y ANCAP ser los instrumentos de aplicación de las políticas, reforzando la vinculación entre ellas y el ejecutivo. Impulsa la creación de fondos sectoriales para financiar la investigación y el desarrollo en el sector. El eje de la oferta se centra en la propuesta de diversificación de la matriz energética y la inclusión de fuentes renovables, la ampliación de las redes de transmisión, y de trabajos permanentes de prospectiva energética. El eje de la demanda se centra en el establecimiento de políticas y normativas financieras de promoción de la eficiencia 
energética. Finalmente, el eje social se enfoca sobre la universalización del acceso a la energía, como derecho humano fundamental.

Este documento fue reforzado con la Ley de Presupuesto 2010 (ley 18.719) que legisla específicamente sobre las obligaciones de la Dirección de Energía DNE y de la Unidad Regulatoria URSEA. Pero quizás lo más importante, es que hubo en el año 2010 un amplio espaldarazo político al crearse la Multipartidaria de Energía, que mediante la formulación del Acuerdo Nacional de Energía en donde participaron todos los partidos con representación parlamentaria, acordó los lineamientos de la PEU2030 como políticas de estado a largo plazo.

Es en el marco de esta política energética planteada por el gobierno de Tabaré Vázquez a partir del año 2005, que se abre un período de desarrollo intenso tanto de generación tradicional, con la incorporación urgente de centrales térmicas, como de formas alternativas y renovables como la energía eólica y la solar fotovoltaica. ${ }^{3}$

\section{1 - Las crisis y el proceso de cambio}

La situación energética hacia el año 2005 no era para nada fácil. Por un lado, Uruguay es un país que no produce petróleo ni gas, por lo tanto todo el suministro energético proveniente de fósiles debe importarse al precio internacional, colocando a su economía energética muy dependiente del precio internacional del crudo. Así es que el país sufrió en carne propia las diferentes crisis petroleras, como la de los países árabes entre 1973 y 1985, generada por la decisión de estos países de no vender crudo a los países que apoyaban a Israel en la guerra del Yom Kippur, las revueltas contra el Sha de Irán y la guerra Irán-Irak (U.S. Department of the States: Office of the Historian, 2019). Tras una década y media de relativa tranquilidad, en el inicio del siglo la enorme demanda de China e India hace subir el precio del barril, que luego generaría un pico histórico en el año 2008. A esto hay que sumarle la crisis del gas de principios de siglo con Argentina, que no puede cumplir con los contratos de exportación. De hecho, los tres gasoductos que une a los países estaban utilizados sólo al $5 \%$ de su capacidad (González y Méndez, 2015). Estos vaivenes económicos y geopolíticos ocasionan inestabilidades muy importantes en la economía local.

Otro factor determinante del estado de situación energético hacia el año 2005 es el estado de su generación hídrica, componente muy importante de su matriz eléctrica. Las últimas centrales hidroeléctricas se inauguraron en el año 1979 (Salto Grande, compartida con Argentina y manejada por la Comisión Técnica Mixta de Salto Grande), y 1982 (Constitución, sobre el Río Negro, manejada por UTE). Sumadas a las centrales de UTE existentes sobre el Río Negro, Gabriel Terra y Rincón de Baygorria, aportan un total de 1538 MW de potencia instalada vertido

${ }^{3}$ En este trabajo nos referiremos indistintamente a la energía generada a través de paneles fotovoltaicos como energía solar fotovoltaica o energía solar. Este trabajo no abarca otras formas de generación energética como la energía solar térmica o la energía eléctrica generada a través de motores térmicos solares con ciclo Stirling. 
al sistema interconectado. De esta manera, la capacidad hidráulica había llegado a un techo por las propias condiciones geográficas de penillanura y llanura, no siendo posible la instalación de otras grandes centrales hidroeléctricas.

Los ciclos climáticos también juegan un rol esencial en el desempeño de la generación. Anualmente se producen ciclos de alta y baja de los niveles de los ríos Uruguay y Negro, que ocasionan estacionalidades en la generación hídrica, la cual se suple con generación de centrales térmicas. También hay eventos extraordinarios (como la corriente del niño) que ocasiona niveles excepcionalmente bajos de los ríos, con lo cual esos años el costo de generación sube por la mayor participación de la generación térmica. En particular, los años 1988 y 1989 fueron de una gran crisis hídrica. En este siglo hubo ciclos de baja producción hídrica en 2004, 2006, 2008 y 2012.

En cuanto a la demanda de energía eléctrica, hubo una explosión de consumo en los año '90 del siglo pasado. En la década anterior el consumo eléctrico había aumentado un $31 \%$. En los noventa aumentó un 76\%, y luego en los 2000 aumentó un 40\% (luego de una caída de consumo en 2001-2003 por la crisis argentina, recuperándose a partir de 2004). En los años 2010 el consumo aumentó un 34\% (MIEM-Datos abiertos, 2021). Este salto anómalo en los noventa debido a la explosión de consumo también sobrecargó al sistema eléctrico.

El conjunto de estos factores de presión sobre el sistema implicó que el estado tenga que hacerse cargo de una rápida recuperación en la capacidad de generación y transmisión a partir de 2005, como veremos en los próximos párrafos.

\section{2 - La evolución de la potencia instalada y la generación eléctrica}

En la figura 2 se muestra la potencia instalada por fuente (en el panel superior) y total (en el panel inferior) para el período 1990-2019 (MIEM-Datos abietos, 2021). Vemos que la potencia instalada de origen hídrico aumenta levemente hasta 1996, y luego se mantiene constante hasta el final de la serie, justamente por la incapacidad de generar nuevas centrales. En el caso de la energía de origen fósil vemos un descenso monótono hasta el año 2005, a partir del cual se comienza un proceso de inversión estatal con el fin de recuperar rápidamente a un sistema sobreestresado, para llegar a un equilibrio a partir de ese año en donde salieron de operación algunas centrales y entraron otras al sistema.

En el gráfico de potencia total podemos ver cómo en el período 1990-2005, a pesar de haber aumentado la demanda en un $78 \%$, la potencia instalada se mantuvo constante debido al proceso de desinversión de las políticas llevadas a cabo en el periodo. La capacidad total instalada comienza a recuperarse a partir de 2005, debido a la entrada de generadores térmicos al principio, y luego de la explosión de la generación eólica, biomasa y solar. 
Por el lado de la generación de energía, se muestra en la figura 3 la generación bruta anual por fuente para el periodo 2002-2019 (panel superior) y la generación detallada mensual en un período más acotado, 2010-2021 (panel inferior). En el gráfico anual se puede ver las consecuencias de los ciclos hídricos largos, que ocasionaron máximos históricos con producción plena de energía hídrica (en particular observar que en el año 2002 casi no se generó con otro tipo de insumo), y mínimos en producción hídrica en los años 2004, 2006, 2008, 2012 y 2016. Especialmente en los años 2008 y 2012 se debió suplir esta falta con generación fósil, pero se observa una caída monótona hasta el fin de la serie en la producción con fósiles, producto de la entrada masiva de las alternativas. El gráfico mensual muestra las consecuencias de la estacionalidad de la hídrica y la importancia de conservar potencia instalada fósil en buen estado de mantenimiento para afrontar las bajas esporádicas de generación de otras fuentes. 

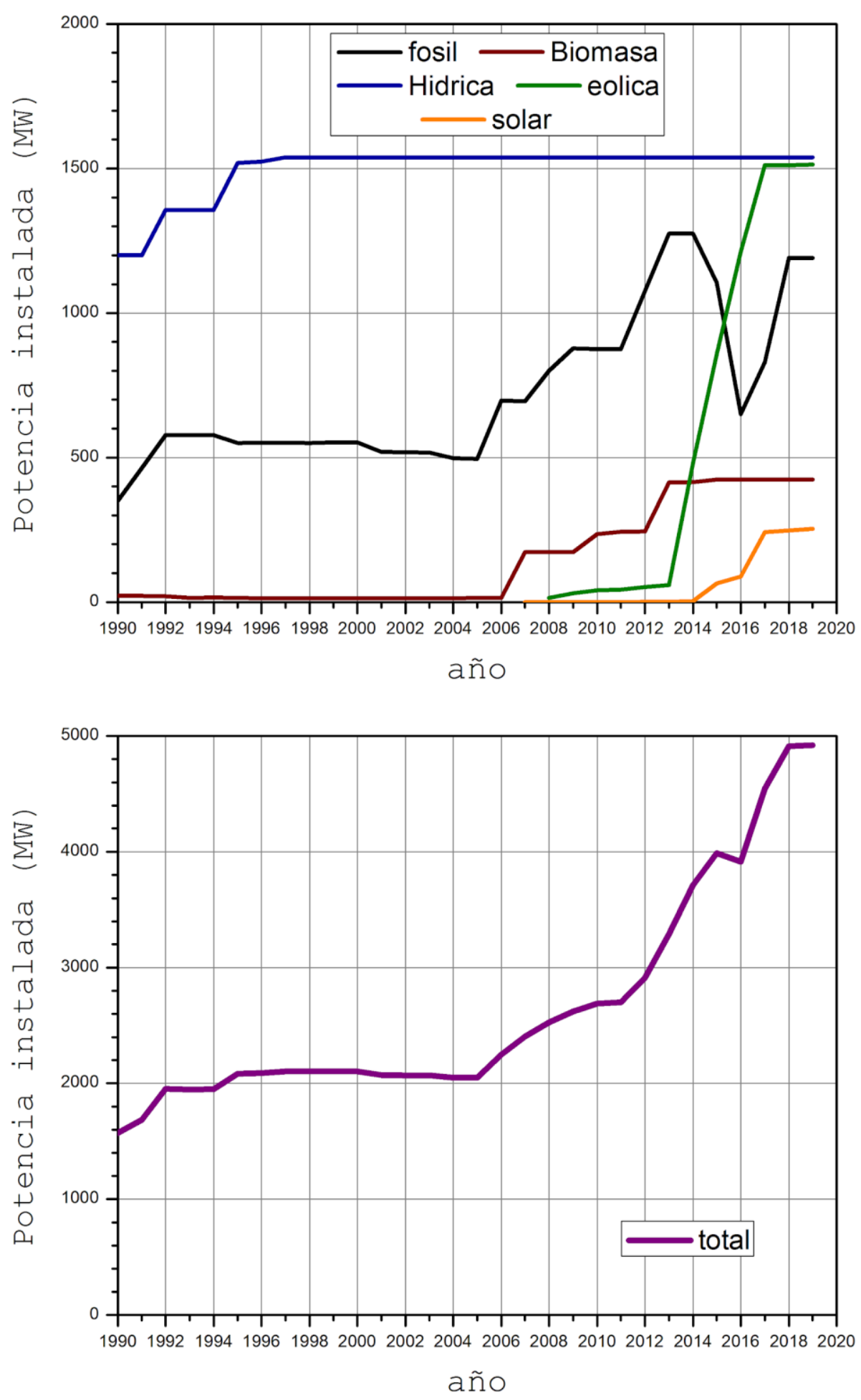

Figura 2: Potencia instalada por fuente (arriba) y total (abajo) 1990-2019. Fuente: elaboración propia en base a datos de MIEM-Datos abiertos (2021). 

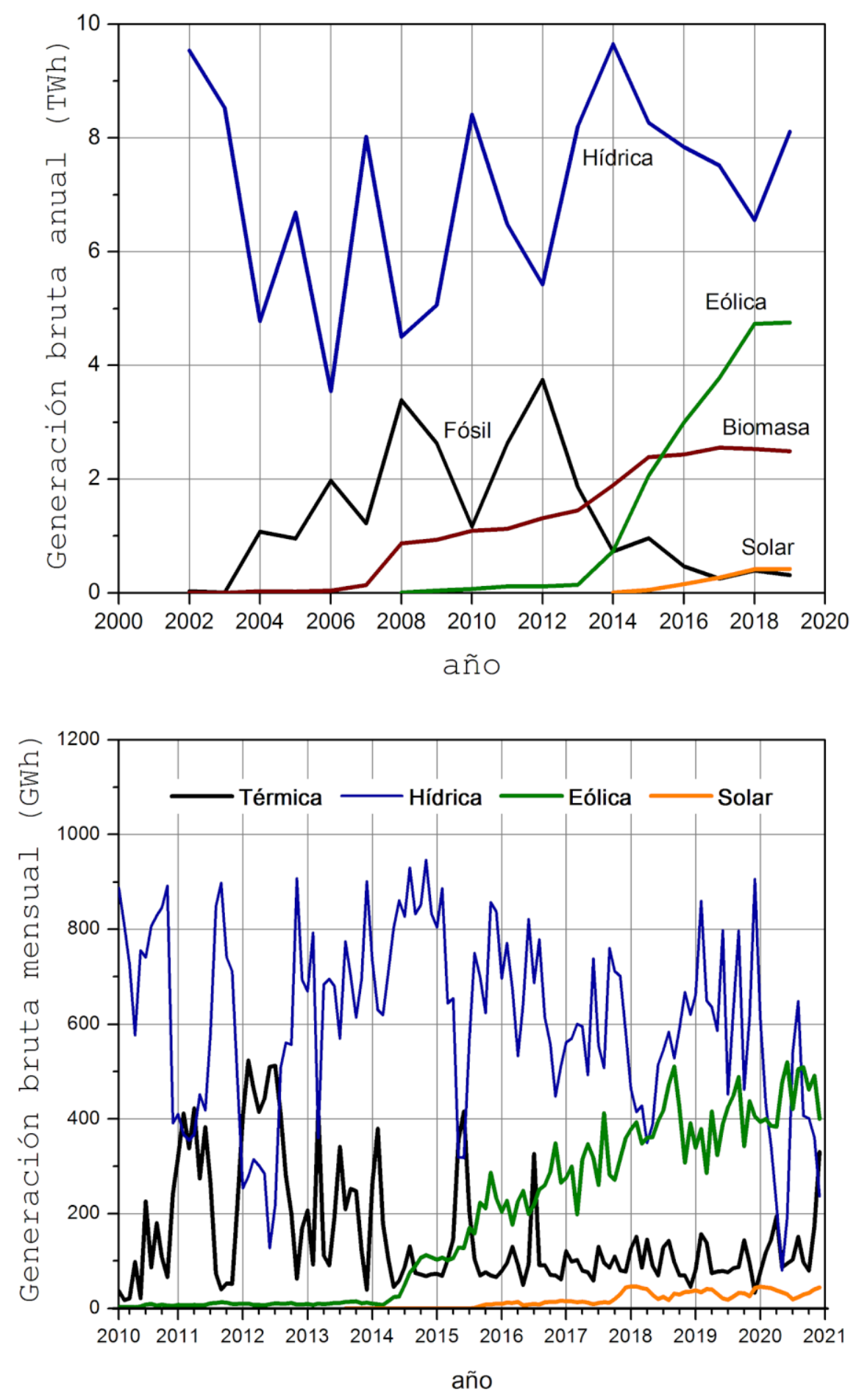

Figura 3: Generación bruta anual 2000-2019 por fuente (arriba). Generación bruta mensual 20102021 por fuente (abajo). En este último gráfico la energía de generación térmica abarca a la fósil y a la biomasa. Fuente: elaboración propia en base a datos de MIEM-Datos abiertos (2021). 


\section{3 - La aparición de generaciones alternativas: la biomasa, la eólica y la solar} fotovoltaica

Si volvemos a la figura 2 podemos apreciar en ella el rápido desarrollo que tuvieron las energías alternativas de producción. La biomasa (aunque ya existía como fuente de generación minoritaria) logra un despegue significativo a partir de 2006, llegando al fin de la serie con un $8,6 \%$ de participación de potencia. La generación eólica logra un despegue desde 2008 para luego escalar con más pendiente desde el año 2014, hasta llegar a un impresionante $30,8 \%$ de participación de potencia (casi igualando a la hídrica). Por el lado de la generación solar fotovoltaica, de crecimiento más atenuado dado que las tecnologías más eficientes recién se desarrollaron a partir del año 2010 debido a un descenso significativo en el costo de generación, comienza a levantarse en el año 2014 hasta llegar a un 5,2\% de participación.

Para la generación proveniente de estas fuentes (figura 3) tenemos un comportamiento similar, creciendo muy fuertemente la generación eólica a partir de 2014, siendo la segunda fuente de generación eléctrica en el Uruguay. Para la energía de origen solar la curva de generación crece más lentamente, pero ya siendo mayor que la generación fósil. También la energía eléctrica de origen térmico a partir de biomasa (principalmente pertenecientes a empresas forestales 0 papeleras), tiene un aumento monótono hasta el fin de la serie.

Todo este proceso de crecimiento representado en estos gráficos, tienen como punto de partida el cambio político del año 2005 , aunque por supuesto los estudios prospectivos y ensayos se venían llevando a cabo desde hace tiempo tanto en la academia como en la empresa de energía UTE. Los primeros estudios de potencial eólico los realizó en los años ‘ 50 del siglo pasado el ingeniero Emanuele Cambilargiu para la Facultad de Ingeniería (FIng) de la Universidad de la República (UdelaR), en la Sierra de los Caracoles (Departamento de Maldonado). Los estudios de potencial eólico de la FIng se hacen más sistemáticos a partir de la década del '90 y confluyen en la instalación de un aerogenerador experimental en la Sierra de los Caracoles, a la vez que se ordena un relevamiento prospectivo del sector energético a la universidad (Nunez y Cataldo, 2005). El prototipo de molino, de potencia $150 \mathrm{~kW}$, fue resultado de la cooperación entre la universidad, el Consejo Nacional de Innovación, Ciencia y Tecnología (CONICyT), la empresa UTE y el financiamiento del Banco Interamericano de Desarrollo (BID) (DNE-DINAMA, 2013).

En el caso de la energía solar fotovoltaica, su desarrollo comenzó más tarde pues como dijimos, dicha fuente energética no resultó competitiva hasta tiempos más recientes con los avances en física del estado sólido que lograron crear celdas mucho más eficientes. En el año 2008 se crea la Mesa Solar, un instrumento del ámbito público-privado que tuvo por objetivo el impulso de la energía solar en el Uruguay con ayuda de fondos de cooperación internacional. Ese mismo año la Facultad de Ingeniería realiza un relevamiento completo del recurso solar en el territorio y publica al año siguiente el mapa solar del Uruguay. En 2010 se crea la Cámara Solar que agrupa a las empresas del sector y se crea el Programa de Energía Solar dentro de la 
Dirección Nacional de Energía (DNE-DINAMA, 2013). La primera planta piloto de generación solar fotovoltaica de $500 \mathrm{~kW}$ (pico), fue inaugurada en Salto en el año 2013, a través de un acuerdo de donación entre el Gobierno del Uruguay y JICA (Agencia Internacional de Cooperación Japonesa). UTE, responsable de la operación y mantenimiento de la planta, participó activamente como contraparte técnica en coordinación con el MIEM, resultando en un proceso de aprendizaje significativo para ambas organizaciones (Energía Solar en Uruguay, 2021). Ese año también comenzaron las primeras licitaciones para la instalación de parques privados.

A pesar de ser una fuente tradicional de generación, la biomasa entra fuerte en el mercado eléctrico a partir del año 2006. Se llama biomasa a los restos de material biológico (en general plantas o derivados), los cuales pueden ser utilizados en forma directa o en forma de biocombustibles. Normalmente provienen de subproductos industriales de industrias forestales, agroalimentarias o del tratamiento de residuos sólidos. Estas industrias son incentivadas a generar electricidad mediante usinas térmicas que aprovechen estos recursos e inyectar energía en el sistema interconectado. Las plantas operativas más importantes entraron al mercado en 2007 (UPM) y 2014 (Punta Pereyra). El proyecto PROBIO del año 2013 se encarga de definir escenarios sustentables de aprovechamiento a gran escala del recurso biomásico de la industria forestal y agroalimentaria. El mismo es una iniciativa conjunta entre el Gobierno Uruguayo y el Programa de las Naciones Unidas Para el Desarrollo, y es financiado por el Fondo Global para el Desarrollo GEF (PROBIO, 2021).

Cabe dedicar aquí un párrafo a la discusión uruguaya respecto de la generación nuclear. Si bien hubo algunos avances en la utilización de un reactor nuclear comprado en 1965 y que fuera apagado en 1985 debido a problemas de corrosión, no fue hasta 1988 que se intensificó la discusión sobre la incorporación de generación nuclear. En ese momento, y debido a la oposición ciudadana en vistas del cercano accidente de Chernobyl, se dejó de lado el proyecto. Más tarde, en 1997, la Ley de Marco Regulatorio del Sector Eléctrico prohibió taxativamente la generación de energía de origen nuclear. Sin embargo, desde el año 2006 se comienza nuevamente a explorar el tema mediante diferentes estudios prospectivos, e incluso se conformó una comisión multipartidaria que evaluaría la factibilidad de la instalación de una central de generación nuclear (Honty, 2011). A este momento, si bien no se ha desarrollado esta posibilidad de generación, sigue siendo un tema en estudio en el país.

Actualmente, existen 43 parques eólicos de los cuales 3 son propiedad de UTE y 5 de UTE con accionistas, y el resto son parques privados. Se instalaron 19 granjas solares, tres de las cuales (de muy baja potencia) pertenecen a UTE y al Ministerio de Energía, el resto son granjas privadas. Esto complementa a las tres centrales hídricas de UTE, la central binacional de Salto Grande co-propiedad con Argentina, las siete centrales térmicas (todas de UTE), y las 11 centrales de biomasa, entre las que se encuentran las de mayor tamaño de las empresas de celulosa: UPM y Punta Pereyra (UTE-Generación, 2021). 


\section{4 - Alianzas conformadas en el proceso de desarrollo}

Con este escenario de desarrollo, hemos armado un mapa conceptual que muestra la configuración de alianzas que permiten el cambio en la matriz eléctrica desde el año 2005 (Figura 4).

En principio, hay un conjunto de factores socio-políticos y naturales que conforman una alianza de desestabilización del sistema eléctrico pre-existente al 2005. La organización deficiente del sistema en cuanto al manejo de las empresas públicas de energía y su coordinación con el ejecutivo mediante la DNE y la ausencia de políticas de manejo eficiente de los recursos es uno de esos factores, quizás el principal. Asociado a eso está el proceso de desinversión de los años 1990 que planchó la potencia instalada en años en donde la demanda energética creció como nunca antes. La falta de provisión del gas argentino influyó también en la generación eléctrica. El precio del barril influye en el costo de generación por fósiles de manera directa, al no poseer el Uruguay acceso local al recurso. Finalmente el factor climático y los ciclos de sequías y bajas precipitaciones opera también sobre un sistema con la capacidad hídrica al límite. Las crisis generadas por estos factores de presión ocasionan restricciones al suministro normal y altos costos. Este panorama es el que toma el gobierno del Frente Amplio a partir del año 2005. 


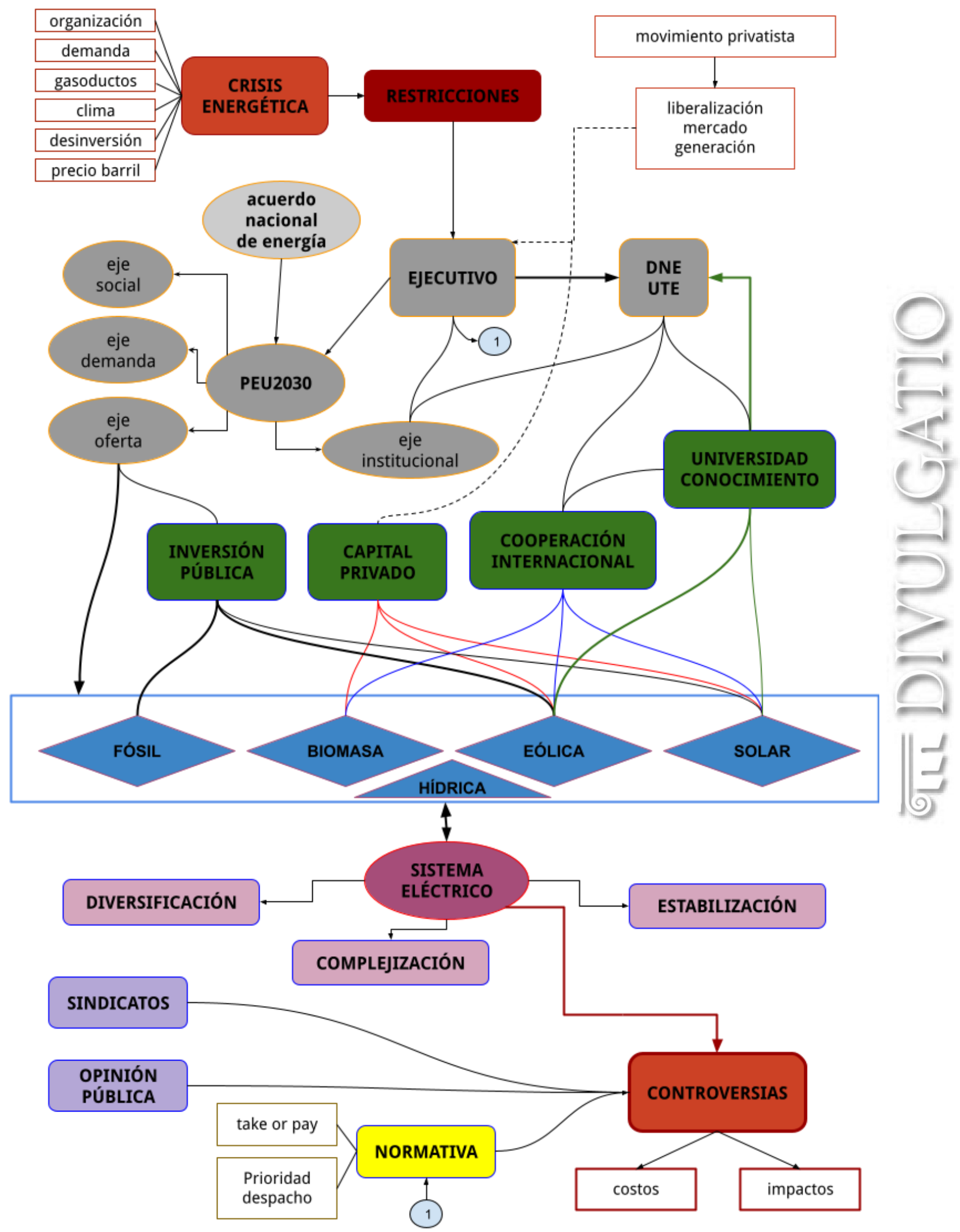

Figura 4: Configuración de alianzas en el proceso de transformación de la matriz eléctrica uruguaya (elaboración propia). 
Ante este escenario, el ejecutivo actúa en forma rápida generando su política energética, PEU2030, en donde alinea cuatro ejes principales de desarrollo. El eje social, estableciendo el derecho al acceso universal a la energía y promoviendo la generalización de la disponibilidad del tendido eléctrico a todos los rincones del país. El eje de la demanda en donde se articulan políticas de promoción de la eficiencia energética (Ley 18.597 del año 2009). En el eje institucional se posiciona en forma clave el fortalecimiento de las funciones ejecutivas de la DNE, reforzando su planta técnica con recursos humanos provenientes del área del conocimiento, principalmente de la Facultad de Ingeniería de la UdelaR. Se estrechan lazos con las empresas estatales, en este caso UTE, en donde también toman funciones de responsabilidad personal científico proveniente de la universidad. Se crean también fondos sectoriales específicos en la Agencia Nacional de Investigación e Innovación (ANNI, recientemente creada). De esta manera la alianza universidadejecutivo aparece como una de las principales causas del fortalecimiento de las capacidades técnicas de la DNE y UTE, al dotar al sistema de recursos humanos altamente calificados en la gestión energética, y se posiciona como el principal traccionante del proceso de desarrollo y diversificación (González y Méndez, 2015).

Por el lado de la oferta, rápidamente se actúa para resolver el cuello de botella de la capacidad productiva estancada, inyectando potencia fósil al sistema con fondos públicos. Se comienza a establecer la necesidad de nuevas fuentes de generación, para lo cual se traza un plan de incorporación de potencia eólica, solar y térmica a partir de biomasa. El mapa de la figura 4 muestra cómo se tejen las alianzas entre la inversión pública, la entrada de capital privado en la generación, los organismos de cooperación internacional y el área del conocimiento científico representado por la universidad, el CONICyT y la ANNI. La inversión pública entró en juego fuertemente en la inyección fósil, como hemos mencionado, pero también en las otras fuentes, principalmente en la eólica con el desarrollo del aerogenerador experimental y la puesta en funcionamiento de los parques de propiedad de UTE y en co-propiedad con privados. En menor medida se establecieron dos pequeñas plantas piloto solares. El capital privado entra en juego fuertemente en la eólica (40 parques de gran porte), en la biomasa (11 centrales térmicas con este combustible), y en menor medida en la solar con 15 plantas.

La cooperación internacional en forma de mecanismos de promoción, fuentes de recursos para investigación y desarrollo y financiamiento directo juega un factor importante en la biomasa, la eólica y la solar. Sólo como ejemplo mencionamos el aerogenerador experimental fue apoyado con fondos del Banco Mundial, las plantas piloto de energía solar fotovoltaica se instalaron con apoyo del gobierno de Japón, y el estudio de las fuentes de biomasa tuvo el apoyo de la GEF. Los estudios prospectivos y de potencial de recurso tanto para eólica como para solar fueron realizados principalmente por la universidad, así como diferentes desarrollos técnicos en plantas piloto. Así, se desarrolla un entramado entre estos cuatro factores: inversión pública, capital privado, cooperación y conocimiento, que es la alianza que expande la capacidad del sistema, que es a su vez fuertemente traccionada por las políticas bajadas del ejecutivo que fueron 
convalidadas por el acuerdo nacional de energía en el parlamento, otorgándoles carácter de políticas públicas estratégicas de largo plazo.

Este proceso tiene como resultado una diversificación y una estabilización del sistema, en cuanto a disponibilidad y variedad de recursos. También resulta en una complejización en la operación técnica y económica, al incrementar su participación las fuentes intermitentes (solar y eólica) cuyos recursos no se pueden regular o reservar ya que dependen de las condiciones atmosféricas y climáticas en ciclos diarios. La participación de más de 60 empresas generadoras implica un desafío en la organización financiera del sistema.

La entrada de estas empresas también está ligada a la integración en la alianza de normativas anteriores al 2005, especialmente la liberalización del mercado de generación. Se le suman normativas propias dictadas por el ejecutivo como el decreto de prioridad de despacho de la eólica (403/2009) por el cual se da prioridad a esta fuente de generación sobre las otras, y principalmente el decreto conocido en la jerga energética como Take or Pay (59/2015), que establece pagar a los operadores de plantas solares y eólicas por la energía que están en condiciones de inyectar en la red pero que por razones de despacho no es inyectada. Esto hace que el estado pague por energía no consumida. Las consecuencias de estos mecanismos de fomento de la inversión ocasionan una percepción de que el estado está pagando por energía no consumida a privados, y con esto el costo de la energía se aumenta para los ciudadanos, lo que genera controversias que analizaremos en la sección 6.

\section{5 - Interpretaciones}

Los factores desestabilizantes que se mostraron al inicio de la sección 4 indudablemente visibilizaron el sistema eléctrico en el marco de los temas públicos. El sistema tantas veces invisible se convirtió en un tema prioritario y esto hizo que el estado tome las riendas y guíe el proceso de diversificación y desarrollo del sistema energético del país, en particular de las fuentes de generación eléctrica.

El cambio de paradigma en las políticas públicas introdujo también un cambio en las interpretaciones imperantes. La flexibilidad interpretativa en cuanto al sistema eléctrico puede ser analizada mediante la multiplicidad de visiones que se desarrollaron antes: científica, económica, ecológica, bienestar social y seguridad. La transición se realiza desde una visión netamente económica de la energía eléctrica, sin demasiados espacios para un manejo político de los recursos y del sistema (visión de seguridad) y con un marginal (pero importante) aporte del sistema de conocimiento (visión científica). Dicha transición propicia las visiones de seguridad al promover un rápido crecimiento de la potencia ofrecida, la visión de bienestar al promover el acceso universal a la energía, de la visión ecológica al promover fuentes de generación renovables, y de la visión científica al promover la integración del sistema científico dentro de la conducción energética. 
La flexibilización de interpretaciones trae también aparejada un escenario de controversias, especialmente entre los que ven a un sistema rico en energía y con exceso de fuentes de generación, incluso con la posibilidad de exportar el recurso, y los que se centran en los costos y los impactos sociales y ambientales del desarrollo. En la próxima sección desarrollaremos estos aspectos controvertidos.

\section{6 - Controversias}

Quizás una controversia menor en cuanto al proceso de diversificación y la entrada en juego de energías alternativas se produce en el campo de los impactos ambientales. En este caso parece ser una controversia que rápidamente se cierra en torno al beneficio que trae aparejado la menor liberación de gases de efecto invernadero de estas tecnologías con respecto a la térmica de origen fósil. Incluso algunas organizaciones ambientalistas consultadas no exhibieron preocupación por impactos ambientales concretos en la instalación de aerogeneradores y parques eólicos de gran porte. ${ }^{4}$ Las empresas que utilizan biomasa, por otra parte, sí están fuertemente cuestionadas pero no por la utilización de subproductos en la generación eléctrica sino por su actividad principal. En general se considera la utilización de biomasa como neutra desde el punto de vista ecológico (PROBIO, 2021). Sólo algunos grupos de vecinos a los parques eólicos expresan sus temores por el impacto visual, ecológico y patrimonial. Por ejemplo, ante la posible instalación de un parque eólico en Sierra de la Ánimas, una comisión de vecinos expresó que “... la construcción del proyecto Río Mirador, que se ubicaría en la zona de Abra del Betete en la Sierra de las Ánimas, afectaría seriamente el ecosistema de esta zona tan característica de nuestro país.." (EL DIARIO, 2013). Agregan en otra nota:

“...el lugar es una de las zonas más emblemáticas del país para todos los uruguayos, sitio de enorme patrimonio natural, científico y cultural... Las Sierras de las Ánimas es de las pocas que hasta el momento se han mantenido libre de aerogeneradores en Maldonado, conservando su carácter natural, con bajísimos índices de elementos antrópicos y una vegetación muy bien conservada..." (UY.PRESS, 2013).

Estas expresiones son muy minoritarias, por lo tanto la interpretación dominante acepta la instalación de nuevas fuentes de generación.

La mayor controversia se centra en torno a los costos y disponibilidad de la energía en el país. Está claro que tomando la visión de la energía eléctrica como una commodity, el Uruguay es un país rico en energía. La responsable de la Dirección Nacional de Energía en el año 2017, Olga Otegui, estimó que con el panorama hasta entonces no se necesitaría la incorporación de nuevos generadores hasta 2023 ya que se posee capacidad instalada muy superior a la demanda

4 Sociedad Amigos del Viento Meteorología Ambiente y Desarrollo, Red Uruguaya ONG's Ambientalistas, y Centro Interdisciplinario de Estudios sobre el Desarrollo. 
(Otegui, 2017). Esta es la interpretación que domina en las áreas de conducción de la energía en el país.
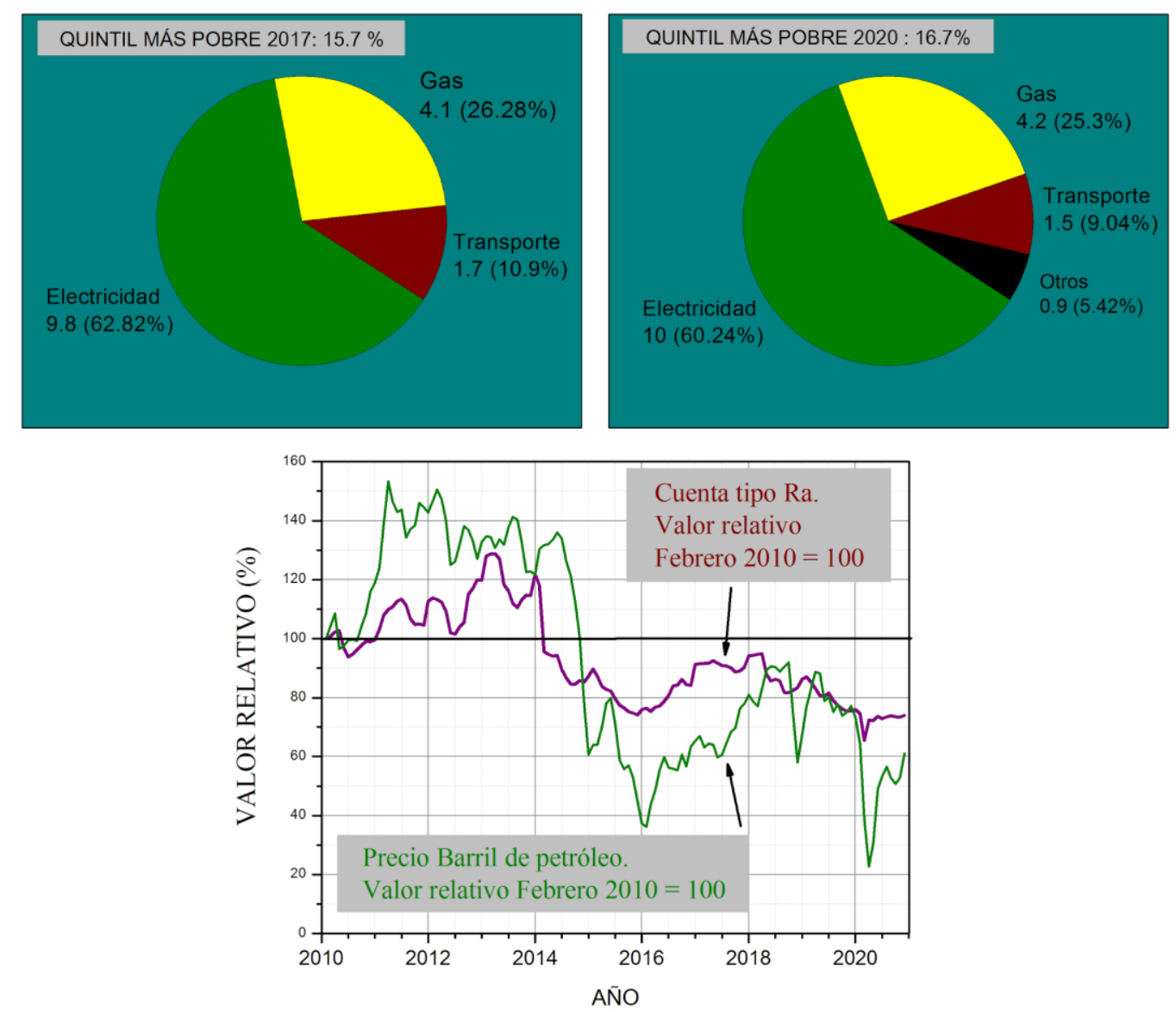

Figura 6: Arriba: Participación del gasto en energía como porcentaje del gasto total del hogar para el quintil más pobre en los años 2017 y 2020. Abajo: Variación del precio residencial de energía dolarizado (Cuenta tipo Ra, Consumo Básico Residencial) y precio del barril de petróleo, ambos relativos a Febrero 2010. Fuente: elaboración propia en base a datos de Jiménez y Yepes-García (2017); Jiménez y Yepes-García (2020); US Energy Information Administration (2021); MIEM-Datos abiertos (2021).

Se abren también otras interpretaciones que consideran la visión de bienestar social, respecto al acceso al suministro y el costo elevado del mismo. Se impone una preocupación por lo que el país paga por energía no consumida debido al decreto take or pay. Se estima por ejemplo que se pagó unos 58 millones de dólares a los generadores por energía que no fue inyectada a la red en el año 2017 (EL OBSERVADOR, 2018). Por otro lado, el país ofrece los índices más altos en la región de porcentaje de ingresos que la población destina a pagar el acceso a la energía. En la figura 6 (paneles superiores) podemos observar el porcentaje de gasto en energía en un hogar uruguayo comparado con sus gastos totales, para el quintil más pobre para el año 2017 (izquierda) y el año 2020 (derecha). Se aprecia en el año 2017 que el quintil más bajo destina 
un $15.6 \%$ de su gasto total a energías, de los cuales casi un $63 \%$ corresponde a gastos de electricidad, un $26 \%$ en gas y un $11 \%$ en transporte. Para el quintil más alto (no mostrado) la participación total del gasto energético es de un 10\% aproximadamente, de los cuales la electricidad se lleva un $46 \%$, el transporte un $43 \%$ y el gas un $11 \%$ (Jiménez y Yepes-García, 2017).

Para el quintil más bajo, el promedio de los países de la región está en $8.4 \%$, superando Uruguay este promedio por un $85 \%$. La participación del gasto en electricidad de la región promediada es del $4.2 \%$, siendo exactamente el doble en el Uruguay. Se suman entonces tres factores que aportan a la controversia. Uno es el alto porcentaje de gasto en energía de su población, en especial la de más bajos ingresos. El otro factor es la preponderancia del gasto eléctrico sobre las demás formas energéticas. La tercera es la desigualdad en el porcentaje de gasto del quintil más pobre sobre el quintil más rico (16\% contra $10 \%$, aproximadamente), desigualdad mayor que el promedio regional. Si comparamos con el año 2020 (figura 6, cuadro superior derecho) el porcentaje de gasto energético sobre gasto total ha aumentado un punto porcentual (hasta 16.7\%), sin embargo el gasto en electricidad se mantuvo estable en alrededor de 10\% (Jiménez y Yepes-García, 2020).

En la figura 6 (panel inferior) comparamos la variación en la década pasada del precio de la tarifa residencial eléctrica (cuenta tipo Ra, tarifa de consumo básico residencial, MIEM-datos, 2021) dolarizada y expresada relativa a la tarifa de Febrero de 2010, con la variación del precio del barril de petróleo también relativa al valor al mismo mes (US Energy Information Administration, 2021). Se observa que el precio de la energía eléctrica residencial copia aproximadamente las variaciones del precio del petróleo, e incluso desde 2016 no ha bajado tanto como el combustible crudo. El descenso desde 2014 se recupera hacia 2018 con algunas variaciones en alrededor del $80 \%$ de su valor de inicios de 2010, para luego caer levemente. Igualmente, estas variaciones al parecer no mejoran la relación entre el gasto eléctrico y el gasto total de los hogares, como se vio anteriormente, tendiendo a mantenerse en un nivel muy alto, el más alto entre los países de la región.

Una de las voces que advierte constantemente sobre esta desigualdad y el alto costo energético es la del sindicato de trabajadores de UTE (AUTE, agrupación UTE). En un comunicado del año 2018 expresan claramente que “... los hogares pagan mucho para garantizar que los Medianos y Grandes Consumidores de energía eléctrica paguen poco, y para que los Generadores Privados de Energía se sigan enriqueciendo con el negocio del cambio de la matriz energética..." (AUTE, 2018). De este modo denuncian el negocio del cambio de matriz en beneficio de los generadores, a los cuales se les paga con contratos a 20 años incluso por la energía no inyectada a la red con el objeto de asegurar la inversión privada, con el consecuente aumento del gasto estatal en energía y el aumento de tarifas. 
Por el contrario, el punto de vista institucional orgánico de la empresa de energía estatal es el contrario. Gonzalo Casaravilla (presidente de UTE hacia 2016) asegura que entre 2010 y fines de 2016, la energía eléctrica "en el bolsillo de la gente" habrá bajado $16 \%$ respecto del índice de precios al consumidor y $31 \%$ en relación a la media de los salarios, gracias al cambio de la matriz de generación (Portal Presidencia Uruguay, 2016). En la nota citada agrega:

“...Nosotros venimos paulatinamente teniendo energía más barata y la estamos suministrando sin dificultad, pero además con perspectiva que Uruguay va a ver energía competitiva que va a permitir que se instale quien quiera en un ambiente de energía que lo permita..." (Portal Presidencia Uruguay, 2016).

Vemos aquí un punto de vista que contrasta claramente con la mirada del sindicato de trabajadores de UTE y de una parte de los analistas energéticos.

En este caso, estamos ante la presencia de una controversia evidentemente no cerrada, pero no nueva en el Uruguay, en donde el costo de la electricidad y en general de los energéticos ha sido históricamente elevado. Las políticas energéticas implementadas y la diversificación de la matriz no trajeron aparejada una mejora sustancial de esta situación, generando entonces dos posiciones bien marcadas entre las cuales transcurre el funcionamiento de la generación eléctrica en el Uruguay: la de un país rico en energía y fuentes de generación alternativa, y la de un país con una pobreza energética no resuelta, entendiendo a ésta como el alto costo en el acceso a la energía para la ciudadanía.

\section{7 - Conclusiones}

Hemos querido sintetizar en este trabajo un proceso de desarrollo y diversificación muy complejo que ha tenido lugar en el Uruguay desde el año 2005, internándonos en diferentes aspectos socio-técnicos para intentar abrir el entramado de factores heterogéneos que lo viabilizaron.

A partir del cambio de gobierno, claramente hubo una posición predominante del ejecutivo como conductor de las políticas públicas, que guió el proceso de desarrollo en alianza con un sistema de conocimiento que pudo dotarlo de recursos humanos altamente calificados que tomaron parte esencial en la conducción energética. De esta manera se formularon políticas delineadas para hacer frente a la compleja situación en la que se encontraba el país, y desarrollar un sistema eléctrico diversificado y estabilizado, aunque la complejidad alcanzada ofrece desafíos en el manejo de un sistema fuertemente alimentado por fuentes intermitentes y una alta heterogeneidad financiera en su composición. La entrada de fondos públicos, capitales privados, fuentes de financiamiento y promoción de organismos de cooperación y la aplicación del conocimiento científico se entreteje en las diferentes fuentes de generación, construyendo un sistema complejo que incluso es considerado sobredimensionado para muchos actores. Las 
visiones contrapuestas de un sistema rico en energía pero una sociedad con el mayor gasto energético por hogar de la región revela controversias aún hoy no cerradas.

Resta saber si en los próximos años Uruguay pueda llegar a una clausura de estas controversias, con una baja sustancial en el gasto energético familiar y manteniendo al sistema activo mediante la inyección de inversión pública y privada, o si se mantendrá el estado de situación en un equilibrio metaestable de larga duración, soportando las diferentes tensiones internas que se hacen evidente en este escrito.

\section{Referencias bibliográficas}

Ardanche, M. (2016). La transformación de la matriz energética en uruguay con énfasis en las energías renovables a partir del año 2005: sobre el progresivo cambio de la configuración material cognitiva o sobre la importancia de los quijotes. Estudio de caso- Red CYTED, 1-24.

Ardanche, M., Bianco, M., Cohano, C., Contreras, S., Goñi, M., Simon, L., y Sutz, J. (2017a). Diálogos, confianzas y aprendizajes para la construcción de políticas de ciencia, tecnología e innovación: La energía eólica en Uruguay. XVII Congreso Latino-Iberoamericano de Gestión Tecnológica.

Ardanche, M., Bianco, M., Cohano, C., Contreras, S., Goñii, M., Simon, L., y Sutz, J. (2017b). The power of wind: An analysis of a uruguayan dialogue regarding an energy policy. Science and Public Policy, 45 (3), 351-360.

AUTE (2017). Comunicado del 9 de Diciembre de 2017. Recuperado 20/2/2021, http://www.aute.org.uy/actividades/noticias/316-la-tarifa-electrica-es-absolutamente-injusta.html Bernardi, R., Arimón, L., D’Ambrosio, L., y Carranza, A. (2019). Integración ciencia-política en el desarrollo eólico de Uruguay. ENERLAC, Revista de energía de Latinoamérica y el Caribe, 3 (2), 8-25. Disponible en: http://enerlac.olade.org/index.php/ENERLAC/article/view/103

Buquet, d., y Chasquetti, D. (2005). Elecciones uruguay 2004: descifrando el cambio. Revista ciencia política (Santiago), 25 (2), 143-152.

DNE-DINAMA (2013). Informe: Medio Ambiente y Energía en Uruguay. Recuperado 10/1/2021, https://www.miem.gub.uy/sites/default/files/informe_medio_ambiente_energia.pdf

EL DIARIO (2 de Agosto de 2013). Vecinos rechazan instalación de parque eólico en la Sierra de las Animas.

EL OBSERVADOR. (4 de Enero de 2018). UTE pagó U\$S 58 millones por energía eólica que no utilizó.

Energía Solar en Uruguay (2021). Recuperado 11/1/2021, http://www.energiasolar.gub.uy/index.php/institucional/energia-solar-fotovoltaica 
Gonzalez, R., y Mendez, R. (2015). Análisis de la elaboración e implementación de la política energética Uruguay 2030. Facultad de Ingeniería, Universidad de la República.

Honty, G. (2011). Energía nuclear en América Latina: el día después. Nueva Sociedad, (234), 32. Hughes, T. P. (1986). The seamless web: technology, science, etcetera, etcetera. Social Studies of Science, 16 (2), 281-292.

IRENA. (2016a). International renewable energy agency. Recuperado 20/6/2016, http://www.irena.org/

IRENA. (2016b). Renewable Energy Policy Brief. Uruguay. International Renewable Energy Agency. Recuperado 20/6/2016,

http://www.irena.org/DocumentDownloads/Publications/IRENA_RE_Latin_America_Policies_201

5_Country_Uruguay.pdf

Jiménez, R. y Yépez-García, A. (2017). Understanding the Drivers of Household Energy Spending: Micro Evidence for Latin America. Banco Interamericano de Desarrollo.

Jiménez, R. y Yépez-García, A. (2020). ¿Cómo consumen energía los hogares?: evidencia de América Latina y el Caribe. Banco Interamericano de Desarrollo.

Jorge, G. (2020). Energías renovables en América Latina: análisis socio-técnico del desarrollo de la energía eólica en el Uruguay. Tesis de Maestría, Universidad Nacional de Quilmes.

Méndez, R. (2008). Informe final de la consultoría sobre energía en el marco del plan estratégico nacional en ciencia tecnología e innovación. PENCTI.

MIEM-Datos abiertos (2021). Recuperado 15/1/2021, https://www.gub.uy/ministerio-industriaenergia-mineria/datos-y-estadisticas/datos

MIEM-Normativa (2021). Recuperado 15/1/2021,

https://www.gub.uy/ministerio-industria-energia-mineria/institucional/normativa

Nunes, V., y Cataldo, J. (2005). Prospectiva tecnológica 2015 en el área energía: Nuevo análisis en la situación actual de crisis energética.

Otegui, M. (2017). entrevistada en: El fenomenal impulso de la energía eólica en Uruguay ahora requiere del usuario. La diaria, 7 de Mayo de 2017.

Pinch, T. J., y Bijker, W. E. (2008). La construcción social de hechos y de artefactos: o acerca de cómo la sociología de la ciencia y la socióloga de la tecnología pueden beneficiarse mutuamente. Actos, actores y artefactos: sociología de la tecnología, 19-62.

Portal Presidencia Uruguay (14 de Junio de 2016). Casaravilla: Precio real de electricidad bajó y es similar al de Chile, Colombia y Brasil. Recuperado 30/4/2021,

https://www.presidencia.gub.uy/comunicacion/comunicacionnoticias/casaravilla-ajuste-costoenergia

PROBIO (2021). Recuperado 11/1/2021, http://www.probio.dne.gub.uy/cms/index.php/generacion/plantas-de-operacion 
Sovacool, B. K. (2009). Rejecting renewables: The socio-technical impediments to renewable electricity in the United States. Energy Policy, 37 (11), 4500-4513.

US Energy Information Administration (2021). Imported Crude Oil Prices. Recuperado 5/1/2021, https://www.eia.gov/outlooks/steo/realprices/

U.S. Department of the States: Office of the Historian. (2019). Oil Embargo, 1973-1974.

Descargado 25/7/2019, de https://history.state.gov/milestones/1969-1976/oil-embargo

UTE-Generación (2021). Recuperado 18/1/2021,

https://portal.ute.com.uy/institucional/infraestructura/fuentes-de-generacion

UY.PRESS. (31 de Julio de 2013). Vecinos rechazan parque eólico en Maldonado. 